\title{
¿ES EL REALISTA CIENTÍFICO UN REALISTA \\ DE LEYES NATURALES?
}

\section{IS THE SCIENTIFIC REALIST A REALIST \\ ABOUT LAWS OF NATURE?}

\author{
Edgar Eduardo Rojas DuRÁN* \\ Universidad Autónoma de Querétaro
}

Resumen: En este artículo se argumenta que, si uno ya es partidario del realismo científico, entonces uno sería también realista de las leyes naturales. Para mostrar esto, se comienza argumentando que sólo los realistas científicos aceptarían que las regularidades no accidentales requieren explicación y que su genuina explicación está dada por leyes naturales. Luego, se arguye que a partir de esto es que los realistas científicos estarían justificados en creer que las leyes naturales existen objetivamente, es decir, son parte de la estructura del mundo físico. Si esto último es cierto, entonces concluimos que parece no haber razones para que los partidarios del realismo científico sean escépticos de la existencia de las leyes naturales.

Palabras clave: realismo científico, antirrealismo, regularidades, explicación, hechos brutos.

АвSTRACT: In this paper, I argue that if one is already an advocate of scientific realism, then one would be also a realist about laws of nature. To show this, I argue that only scientific realists would accept that non-accidental regularities require explanation and that their genuine explanation is given by laws of nature. Then, from this conclusion, it seems that scientific realists have reason to believe that there are laws of nature in an objective sense. If this is correct, the conclusion is that there are no reasons for advocates of scientific realism to be skeptics about laws of nature.

KeYwORDs: scientific realism, anti-realism, regularities, explanation, raw facts.

* Postdoctoral fellow, 16 de septiembre 57, Centro Histórico, Santiago de Querétaro, Querétaro, 76000, rode8505@gmail.com 


\section{Introducción}

En filosofía de la ciencia hay dos perspectivas o posiciones en torno a las teorías científicas: el realismo científico y el antirrealismo. El realismo científico es la perspectiva según la cual nuestras mejores teorías actuales son descripciones aproximadamente verdaderas del mundo físico tanto en sus aspectos observables como inobservables (Chakravartty 2007, p. 231). En contraposición, el antirrealismo, en general, consiste en la perspectiva que niega esto. Sin embargo, hay varias versiones. Una de ellas es el instrumentalismo. Este tipo de antirrealismo piensa las mejores teorías científicas actuales como meros instrumentos o herramientas conceptuales que no pretenden ser descripciones de entidades ni procesos físicos. Si se piensan así las teorías, entonces queda pendiente una explicación de por qué tales herramientas o artilugios conceptuales tienen el poder predictivo que de hecho tienen. Una explicación que nos quedan a deber los partidarios de esta concepción. Otra de ellas es el empirismo constructivo. Esta posición cuestiona la necesidad de una explicación del éxito de las teorías científicas y sugiere que pensemos en el desarrollo de la actividad científica como un mero fenómeno biológico. En consecuencia, sugiere pensar las teorías científicas actuales como organismos bien adaptados. Así concebidas sólo las teorías mejor adaptadas, es decir, aquellas que gocen de mayor éxito predictivo y explicativo respecto a las demás, actuales o pasadas, serán las que sobrevivan, mientras que las demás quedarán en el olvido. (cfr. Van Fraassen 1980, pp. 39-40). Esta forma de antirrealismo ${ }^{1}$ deja pendiente la búsqueda de la explicación de la buena adaptación de las teorías, es decir, su éxito predictivo, pues considera que tal adaptación es un hecho que simplemente sucede y no requiere explicación. Ambas versiones, como se puede apreciar, tienen en común que dejan pendiente de explicación el asunto no menos importante de qué hace que nuestras mejores teorías científicas actuales tengan el éxito predictivo y explicativo que de hecho tienen. Un asunto que el realista científico, en sus diferentes versiones, no soslaya y al cual ofrece una explicación: que muchas de

\footnotetext{
1 Además de caracterizar así el desarrollo científico, el Empirismo Constructivo es la postura que afirma, en contra del realismo científico, que las teorías no son (aproximadamente) verdaderas, sino empiricamente adecuadas y que éstas implican conocimiento sólo del aspecto observable del mundo. Si el partidario de esta posición aceptase que el éxito predictivo y explicativo de las teorías científicas actuales requieriese explicación, lo que explicaría tal es que éstas son empiricamente adecuadas (dan cuenta de los fenómenos) y que las entidades observables que postulan éstas existen objetivamente. Sin embargo, esta posición de entrada es reacia a dar una explicación del éxito de las teorías científicas y sugiere que deberíamos considerarlo como algo que simplemente sucede.
} 
las entidades inobservables descritas de manera putativa por nuestras mejores teorías científicas existen objetivamente (cfr. Chakravartty 2017, p. 68).

Ahora bien, dentro de las teorías científicas pertenecientes a la física, química y biología encontramos el término "ley". En torno a este último hay disputa entre los partidarios del realismo y el antirrealismo también. Esta disputa es un apéndice de la discusión en torno de cómo interpretar nuestras mejores teorías científicas actuales. Los partidarios del realismo de leyes naturales afirman que dicho término refiere a enunciados generales necesariamente verdaderos en virtud de algún tipo de relación o conexión necesaria entre universales (Dretske 1977, Tooley 1977, Armstrong 1983) o entre propiedades de manifestación y estímulo (Bird 2005). Por otra parte, los antirrealistas niegan en general que dicho término refiera a enunciados generales necesariamente verdaderos en virtud de una conexión tal. Un tipo de antirrealismo de leyes naturales afirma que el término "ley natural", aunque sea empleado en el seno de muchas de las teorías científicas para predecir un sin número de hechos, refiere a un enunciado general verdadero en virtud de una conexión contingente entre conjuntos de hechos particulares observados del mismo tipo, es decir, una regularidad (Lewis 1973). Así, esta forma de antirrealismo de leyes naturales es en última instancia una reducción de las leyes naturales a regularidades. Esta reducción se desprende del escepticismo de que haya conexiones necesarias entre conjuntos de hechos observados particulares sucesivos del mismo tipo. Este escepticismo tiene su origen en el empirismo de David Hume, en específico, en la sección VII de "Una investigación concerniente al entendimiento humano". El escepticismo de la idea de conexión necesaria tiene su fundamento en que ésta carece de un fundamento empírico o no proviene de sensación perceptual alguna. Por esta razón es que uno no debería creer en su existencia objetiva.

Otro tipo de antirrealismo de las leyes naturales consiste en la afirmación de que el término "ley natural" refiere a aquellos enunciados generales verdaderos que fungen como axiomas o teoremas dentro del mejor sistema deductivo que nos permita inferir deductivamente la totalidad de los hechos particulares del mundo (la totalidad del mosaico humeano), es decir, la teoría científica, axiomatizable en principio, que nos prediga la totalidad de los hechos y que goce del mejor equilibrio entre simplicidad y fortaleza deductiva. Esta forma de caracterizar las leyes naturales lo encontramos en Lewis (1973) Y ha sido criticada por varios autores, entre ellos Carroll (1994) como incompleta en virtud de que la noción de simplicidad y fortaleza requieren de un sujeto para poder establecer que un sistema es más simple y fuerte que otro. 
Por último, tenemos un tercer tipo de antirrealismo de las leyes naturales. Éste surge como consecuencia natural del ya bien conocido argumento de la meta-inducción pesimista de Laudan (1981) y afirma que, si pensamos en las leyes naturales como constituyentes de la estructura del mundo, entonces carecemos de razones para creer que los enunciados generales verdaderos que los científicos denominan "leyes naturales" de hecho lo sean en virtud de conexiones necesarias inobservables. Esta carencia de razones procede de la historia de la ciencia. En ella encontramos que lo que los científicos denominaban "ley natural" en teorías pasadas, posteriormente se encontró que no lo era en virtud de que su generalidad se vio acotada debido a que se encontraron excepciones a ésta, o se encontraron enunciados verdaderos más generales a partir de los cuales se podrían derivar, en principio.

A continuación, argumentaré, primero, que resultará nada extraño a los realistas que las regularidades interesantes para las ciencias naturales requieren explicación y, segundo, que ésta sólo se obtiene genuinamente mediante leyes naturales. Posteriormente, argüiré que el hecho de que las leyes naturales, caracterizadas como conexiones, vínculos o relaciones necesarias entre universales o propiedades de manifestación y estímulo, expliquen este tipo de regularidades es razón para que los partidarios del realismo científico que creen en la justificación de la inferencia a la mejor explicación en términos confiabilistas crean que éstas existen objetivamente.

Antes de comenzar con el núcleo del trabajo, resulta conveniente resaltar que este manuscrito sigue la misma línea de trabajos anteriores como los de Berenstain y Ladyman (2012), así como los de Borge (2016a, 2016b) en los que se afirma que hay una implicación indirecta entre ser realista acerca de las teorías científicas y ser realista acerca de la modalidad. Sin embargo, mientras que en estos trabajos se arguye que es perfectamente coherente ser realista científico y creer en la existencia de una modalidad objetiva, aquí se arguye de alguna manera y en última instancia que hay una coherencia entre ser realista científico y creer en la existencia objetiva de ciertos elementos que conforman dicha modalidad: las leyes naturales. Esto no quiere decir que las leyes naturales agoten la totalidad de la estructura modal objetiva del mundo. Borge (2016a, 2016b) arguye que el argumento que da el realista científico a favor de la existencia de las entidades que éstas postulan como la mejor explicación de su éxito predictivo y explicativo tiene implicaciones modales cuando uno analiza que se está entendiendo por explicación por parte del realista científico: una explicación metafísica o grounding, así como que la teoría descriptivista-causal, la mejor teoría de la 
referencia que haría sentido para las diversas versiones de realismo científico en cuanto a dar cuenta del progreso científico se refiere en términos de que hay una continuidad referencial en las teorías pasadas y presentes, tiene el mismo tipo de implicaciones. Berenstain y Ladyman (2012), por su parte, sostienen que es compatible ser realista científico, en su versión estructural, y ser un realista acerca de la estructura modal del mundo, así como que dicha estructura modal se compone de leyes naturales, causalidad y probabilidad. En cuanto a las leyes se refiere, éstas no pueden caracterizarse satisfactoriamente en términos de la tesis del mosaico humeano ni en términos del papel que éstas juegan, en tanto enunciados generales verdaderos, dentro de las mejores teorías actuales del mundo. Mi trabajo, por su parte, explora más bien, por un lado, la relación que hay entre los supuestos epistémico-ontológicos que suscriben en el fondo los partidarios del realismo científico en general, lo que Chakravartty (cfr. 2007, p. xiv) denomina "aspectos metafísicos del realismo" y la creencia de que las leyes naturales explican genuinamente las regularidades y, por el otro, el vínculo que hay entre el compromiso realista acerca de la justificación de la inferencia a la mejor explicación en general, compromiso que suscriben los realistas en el fondo cuando afirman que la existencia objetiva de las entidades postuladas por las teorías científicas es la mejor explicación de su éxito predictivo y explicativo, y la justificación para inferir que las leyes naturales existen objetivamente en virtud de que explican genuinamente las regularidades

\section{Desarrollo}

Es un hecho que hay regularidades tales como (a) todas las esferas de oro puro tienen un diámetro menor a 1,609.34 metros y (b) todas las esferas de uranio 235 tienen un diámetro menor a 1,609.34 metros. Este tipo regularidades son de interés para los científicos. Mientras que ningún científico piensa que (a) sea una verdad necesaria, algunos piensan que (b) sí lo es. La razón de esto estriba en que, por un lado, no parece haber ninguna propiedad fundamental en el oro puro que impida que haya esferas con un diámetro mayor a 1,609.34 metros, es decir, es un hecho meramente contingente que no haya una cantidad de oro suficiente para producir alguna vez una esfera de semejante tamaño, mientras que, por el otro, los científicos sí conocen una propiedad del elemento radioactivo Uranio 235 que impide que haya esferas mayores a dicho diámetro: su masa crítica. Por lo tanto, los científicos hacen la distinción entre verdades necesarias, tales como (b), y verdades contingentes, tales como (a). Esta distinción es aceptada por los realistas de leyes naturales, pero no así por los antirrealistas. Esto se debe a que los últimos consideran que a pesar de que los científicos crean que hay 
verdades necesarias como (b), éstas son en el fondo verdades contingentes. Al afirmar esto, los antirrealistas nos deben una explicación de por qué de hecho los científicos hacen esta distinción. Una distinción de suma importancia para el conocimiento científico en la medida en que se asume cuando los científicos nos brindan predicciones de determinados fenómenos en términos de verdades necesarias.

Por otra parte, hay otro tipo de verdades necesarias tales como (c) todos los cuerpos en caída libre se precipitan sobre la superficie terrestre con una aceleración de 9.81 metros por segundo al cuadrado y (d) todos los planetas se mueven en órbitas elípticas con el Sol en uno de los focos. Los científicos saben que este tipo de verdades se dan necesariamente sólo bajo ciertas condiciones bien determinadas. Por esta razón es que a este tipo de verdades se les denomina "leyes ceteris paribus". Este tipo verdades son las que denominaré "regularidades nómicas". Este nombre se debe a que los científicos las denominaron leyes naturales en su momento debido a que tenían buenas razones para creer que eran de hecho genuinas verdades necesarias sin excepciones.

\subsection{Las regularidades nómicas tienen explicación}

El tipo de verdades como (c) y (d), es decir, las regularidades nómicas, tienen explicación. Esta afirmación tiene como supuesto el Principio de Razón Suficiente (PRS) en su versión epistémica. Éste afirma que todo tiene una explicación. Este principio es el que suscriben de hecho los científicos para llevar a cabo sus investigaciones en cualquier ámbito de la naturaleza. Los realistas científicos también suscriben dicho principio pues consideran de suma importancia brindar explicaciones, aunque se tenga que recurrir a entidades o procesos inobservables. Sin embargo, este principio o bien (i) no es suscrito por los empiristas en general o bien (ii) es suscrito de manera parcial. Si no es suscrito, entonces el empirista dejaría sin explicación las regularidades nómicas y argüiría que son hechos brutos. Si es suscrito de manera parcial, entonces el empirista sólo aceptará explicaciones que estén en términos de observables. ¿qué explicación puede satisfacer dicho requisito? Una explicación en términos de regularidades.

Antes de continuar, veamos con más detalle qué implicaría que las regularidades nómicas sean consideradas como hechos brutos o fundamentales en la naturaleza que carecen de explicación. Para este fin exploraré la teoría de hechos brutos de Barnes (1994). Según ésta, hay dos tipos de hechos brutos: 
los epistémicos y los ontológicos. Los primeros tienen de hecho explicación a pesar de que la desconozcamos ahora. Los segundos son aquellos que carecen de explicación en términos distintos a ellos mismos.

Afirmar que $\mathrm{H}$ es un hecho epistémicamente bruto significa que $\mathrm{H}$ carece de explicación en un momento dado, uno que resulta del estado del conocimiento actual. Para ilustrar pongamos como ejemplo la ley de Boyle-Charles. Ésta fue considerada como "hecho bruto" hasta que fue incorporada dentro de la teoría cinemática de los gases y fue explicada por esta última.

Por otra parte, afirmar que $\mathrm{H}$ es un hecho ontológicamente bruto o fundamental significa que no tiene explicación en lo absoluto, sin importar cuando lo afirmemos y por ende sin importar estados de conocimiento subsecuentes. Para Barnes, algunos ejemplos de este tipo de hechos serían las leyes naturales o principios físicos fundamentales tales como el hecho de que el universo tiene una determinada cantidad de masa o energía en vez de otra o el hecho de que el universo existe en lo absoluto. Estos son hechos a partir de los cuales se derivan muchos otros y que son derivados de ningún otro. En este sentido es que se explican apelando a sí mismos, es decir, su base explicativa consiste en ellos mismos.

Esta demarcación entre hechos brutos epistémicos y ontológicos es debatible, puesto que uno podría afirmar como Fahrbach (2005) que lo que hace bruto o fundamental a un determinado hecho es que carece de explicación en lo absoluto. Por lo tanto, no parecería tener sentido afirmar que los hechos brutos tengan como base explicativa a sí mismos, sino más bien que al reconocerlos como tales obtenemos una mejor comprensión de la estructura de nuestro mundo.

Sin embargo, me parece que la distinción hecha por Barnes es pertinente en la medida en que tenemos casos muy bien conocidos de hechos que se consideraron como brutos o fundamentales y que posteriormente se les encontró explicación en términos distintos a ellos mismos. Estas historias muy bien pueden repetirse en lo sucesivo. Por lo tanto, podemos afirmar con seguridad que hay hechos brutos en sentido epistémico y considerar como una posibilidad la existencia de hechos brutos o fundamentales en sentido ontológico, pues algunos de los principios físicos o leyes naturales fundamentales parecen no tener explicación. Ahora bien, cómo caracterizar estos últimos es un asunto que no abordaré aquí y que es motivo de discusión entre Fahrbach y Barnes. 
Expuesto lo anterior, no parece razonable aceptar que las regularidades nómicas sean hechos brutos ontológicos. Considerarlas de esta manera debería ser nuestra última opción. Si los científicos asumiesen que las regularidades nómicas son hechos brutos ontológicos, entonces no buscarían su explicación. $\mathrm{Si}$ no buscasen su explicación, conforme progrese la actividad científica en otros ámbitos encontrarán nuevas regularidades nómicas y, en consecuencia, el conjunto de los hechos brutos ontológicos crecería indefinidamente. Si este conjunto creciese indefinidamente, entonces llegaría un punto en el que la investigación científica se toparía con que hay un número infinitamente grande de leyes o principios fundamentales y tarde que temprano se detendría. Sin embargo, esto no ha sido el caso. El conocimiento científico actual nos dice que hay una cantidad reducida de principios, leyes o constantes fundamentales y la actividad científica sigue en marcha en tanto búsqueda de sus posibles explicaciones. En consecuencia, aceptar que las regularidades son hechos brutos fundamentales, como sugiere el empirismo en primera instancia, llevaría a la consecuencia indeseable de que el conocimiento científico estuviese plagado de leyes o principios fundamentales en cada una de sus ramas o campos de estudio al grado de explicar muy poco y al paro de la investigación científica.

Así, los científicos históricamente han suscrito el PRS cuando se trata de la metodología para la investigación científica: "buscar la causa o explicación de alguna entidad $x$ (leyes naturales o principios fundamentales incluidos) sin asumir que $x$ es un hecho bruto. Esta versión del PRS es un principio metodológico que permite a los científicos desarrollar la investigación científica en cada uno de los campos del conocimiento que trae como resultado nuevas explicaciones incorporadas en nuevas teorías. Si uno no dudase acerca del estatus ontológico de los hechos brutos, dado el conocimiento de fondo en determinado tiempo, carecería de sentido emprender la búsqueda de su explicación o causa (sin importar si de hecho la tiene o no).

Sin embargo, ha sido recurrente pensar que, si aceptamos el PRS, entonces uno se enfrenta con la pregunta de qué explica a este principio. En consecuencia, parece que el PRS abre la puerta a una regresión explicativa. De acuerdo. Sin embargo, esta regresión es una que va de la mano con el desarrollo de las teorías científicas, i.e., con la práctica científica. Pues a pesar de que es verdadero que las explicaciones científicas se detienen en un momento dado dentro de un determinado contexto epistémico o estado del conocimiento, que esto suceda no implica que la explicación hasta ese momento encontrada sea de hecho definitiva o completa, ya que esta podría cambiar en la medida en que 
se convierta en parte derivada de una explicación ulterior más comprensiva. En consecuencia, me parece que lo más prudente es afirmar que desde nuestro estado del conocimiento actual, el PRS parece carecer de explicación o causa, uno simplemente lo considera como intuitivamente correcto y como indispensable para la actividad científica de investigación y para el deseo humano de buscar explicaciones en general.

Por otro lado, cuando se trata de las leyes naturales, la explicación de un determinado fenómeno natural se detiene por un momento, dado el estado actual de conocimiento, cuando se ha enunciado una ley que establece la manera en que tal fenómeno en cuestión (hasta entonces considerado como sin causa aparente) se comporta. Sin embargo, la posibilidad de que haya explicación a esta ley permanece abierta. Puede ser que de hecho la tenga y simplemente la ignoremos o puede ser que no la tenga en lo absoluto. Si o no la tiene es algo que un estado del conocimiento posterior determinará. Por lo tanto, lo más razonable, si uno suscribe el PRS, es considerar a las leyes naturales y principios físicos fundamentales como hechos brutos en sentido epistémico y dejar abierta la posibilidad de que, sin importar cuántos estados del conocimiento pasen, no tengan explicación alguna ${ }^{2}$.

Algunas leyes naturales (consideradas como hechos brutos o fundamentales en sentido epistémico) parecen tener explicación en términos de leyes más generales o principios físicos más fundamentales (considerados como hechos brutos o fundamentales en sentido ontológico). A pesar de que es verdadero que en el momento en que éstas fueron enunciadas pudieron haber sido consideradas como hechos brutos (dado el estado de conocimiento de ese momento), posteriormente se les encontró explicación (dado un estado posterior de conocimiento) y así sucesivamente. Un claro ejemplo de esto lo podemos encontrar si echamos un vistazo a las leyes de Kepler y a la ley de Galileo de los cuerpos en caída libre. Ambas fueron consideradas en su momento como "hechos brutos o fundamentales" (pues parecían carecer de explicación y resultaban, en consecuencia, serios candidatos a serlo en sentido ontológico), pero posteriormente fueron explicados o unificados a través de las leyes del movimiento de Newton y su ley de la gravitación universal. Otro ejemplo tiene

\footnotetext{
${ }^{2}$ Es importante remarcar que Friedman (1974) ya había sugerido anteriormente pensar en las leyes que encontramos en las teorías científicas como hechos brutos cuando intenta dar cuenta de cómo es que las teorías científicas nos brindan entendimiento en virtud de que unifican. Esta misma idea es en esencia mantenida por Kitcher (1981) cuando nos ofrece su versión de la teoría de explicación por unificación en términos de patrones argumentativos.
} 
que ver con la teoría cinemática de los gases. Según Friedman, esta teoría explicó fenómenos que tienen que ver con el comportamiento de los gases integrando hechos considerados como brutos anteriormente: la ley de Graham, la de BoyleCharles y las capacidades de calor específico de los gases, en uno sólo: que las moléculas que integran los gases satisfacen las leyes de la mecánica (Friedman 1974).

\subsection{Las regularidades no son explicadas por sí mismas}

Regresando a la posibilidad de que los empiristas suscriban el PRS de manera parcial o acotada al aceptar como explicación de las regularidades nómicas sólo una que esté en términos de observables, tenemos entonces que la única explicación aceptable para ellos sería en términos de las regularidades mismas. Sin embargo, esta no es una vía posible. Esta imposibilidad se hace patente a través de una pequeña prueba hecha por Bird $(1998$, p. 30) la cual, a su vez, es muy similar a la hecha previamente por Armstrong (1981, pp. 40-41). Ésta requiere dos suposiciones clave. La primera es que una regularidad consiste de todas sus instanciaciones. De esta manera, la regularidad de que los $F$ son $G$ es equivalente a $(\mathrm{Fa} \& \mathrm{Ga}) \&(\mathrm{Fb} \& \mathrm{~Gb}) \& \ldots$. La segunda consiste en que algo no puede explicarse a sí mismo. Esto puede ilustrarse con el hecho de que haya lluvia explica el hecho de que el pavimento esté mojado, pero no explica por qué hay lluvia. Establecidas ambas suposiciones podemos proceder al desarrollo de la prueba de que las regularidades no se explican a sí mismas.

Supóngase que hay una regularidad que los F son G. Supóngase que sólo hay cuatro objetos en el mundo que son Fs: a, b, c y d. El enunciado general 'todos los F son G' es equivalente a: (A) (Fa\&Ga)\&(Fb\&Gb)\&(Fc\&Gc)\&(Fd\&Gd) $\&$ (nada más es $\mathrm{F}$ que $\mathrm{a}, \mathrm{b}, \mathrm{c}$ o d). La conjunción de todas las instanciaciones es: (B) $(\mathrm{Fa} \& \mathrm{Ga}) \&(\mathrm{Fb} \& \mathrm{~Gb}) \&(\mathrm{Fc} \& \mathrm{Gc}) \&(\mathrm{Fd} \& \mathrm{Gd})$. (A) y (B) son idénticos excepto por la última parte del primero. Por lo tanto, llamémoslo (C). (C) nada más es $\mathrm{F}$ que a, b, c o d. En consecuencia, $(\mathrm{A})=(\mathrm{B}) \&(\mathrm{C})$. Si esto es así, entonces decir que (A) explica (B) es lo mismo que decir que (B)\&(C) explica (B). Pero esto último no puede ser el caso dado nuestra segunda suposición clave y el hecho de que (C) no contribuye a explicar por qué los F son G. En consecuencia, nada a lo que se hace referencia en (A) puede explicar por qué es el caso que (B). En otras palabras, cualquier regularidad descrita por cualquier enunciado general no explica sus instanciaciones. En breve, una regularidad no se explica a sí misma. 
Cabe señalar que la prueba aquí presentada no socavaría, en principio, la teoría de regularidades presentada por Psillos (2014). Según ésta, una regularidad consiste en el conjunto de todas sus instanciaciones y algo más: un patrón que las unifica. Sin embargo, examinada de cerca tal manera de caracterizar las regularidades no sería una acorde al empirismo en general, pues al postular patrones unificadores en la naturaleza termina haciendo una tesis metafísica que el empirismo difícilmente aceptaría estamos justificados en hacer. Por lo tanto, la posibilidad de explicar las regularidades apelando a ellas mismas queda excluida, so pena de abandonar el empirismo.

\subsection{Las regularidades son explicadas por las leyes naturales}

Por consiguiente, si las regularidades no se explican a sí mismas, tampoco lo hacen las regularidades nómicas. Si no tenemos explicación de las regularidades nómicas en términos exclusivamente de observables ¿en qué términos sí la tenemos? En términos de inobservables tales como relaciones necesarias entre universales, teoría universalista (Dretske 1977, Tooley 1977, Armstrong 1983), o relaciones necesarias entre propiedades de estímulo y manifestación, teoría disposicionalista (Bird 2005), es decir, leyes naturales ${ }^{3}$. Sin embargo, es pertinente señalar aquí que aparentemente hay una forma de explicar las regularidades en términos de inobservables que no sean leyes naturales. Esta forma es la defendida por Mumford (2004) y consiste en afirmar que las conexiones necesarias son suficientes para explicar las regularidades. Sin embargo, por un lado, las leyes naturales, siguiendo a Bird (2007, p. 204) y su concepción disposicionalista (Bird 2005), están estrechamente ligadas a ellas o en última instancia se identifican con ellas, por el otro, Borge (2015) ha mostrado que los argumentos o razones presentados por Mumford para abrazar su explicación de regularidades en términos de meras potencias y prescindir así de las leyes naturales de nuestro marco ontológico son insuficientes en la medida en que dependen de una noción de "papel gobernante" y supuestos acerca de la sobreviniencia cuestionables. Por lo tanto, la explicación de regularidades en términos de conexiones necesarias y cualquier metafísica disposicionalista se identifica en última instancia con la de leyes naturales, si entendemos por leyes

\footnotetext{
3 Caracterizar así las leyes naturales es la única manera posible de que las regularidades no sean explicadas por sí mismas. Por lo tanto, la caracterización eliminativista de Psillos (2014) no es una posibilidad genuina de explicar las regularidades en tanto ésta piensa las leyes naturales en última instancia como regularidades de cierto tipo: aquellas que tienen determinados patrones unificadores.
} 
naturales verdades generales necesarias en virtud de una conexión, vínculo o relación necesaria entre entre propiedades de estímulo y manifestación (Bird 2005). Asimismo, el tipo de explicación propuesto por Mumford, en tanto pretende excluir las leyes naturales de nuestra ontología, tampoco es una mejor que la dada por la concepción de leyes en términos de una conexión, vínculo o relación inexorable entre universales, en tanto se enfrenta a los problemas que supuestamente trataba de evitar: el quiddismo y apelar a universales (Borge 2015). Sin embargo, esta explicación, en sus dos variantes, apela a entidades inobservables tales como conexiones o relaciones necesarias. Por consiguiente, si uno no privilegia la explicación en términos de observables e inobservables por igual, como lo hacen algunos empiristas, parece que uno se quedaría hasta aquí. Esto, para el realista, conduce a un tipo de insatisfacción, pues no estaría contento con aceptar que tales regularidades son hechos brutos en la medida en que, si no tienen explicación en términos de regularidades observables, entonces no la tienen en lo absoluto. De hecho, la historia de la ciencia nos ha mostrado que los científicos no se contentan con explicaciones de lo observable en términos de observables, han postulado entidades y procesos inobservables como explicaciones de fenómenos observables. De esta manera, los realistas parecen suscribir principios que van de acorde con la investigación científica. Así, la explicación de las regularidades nómicas sólo puede ser en términos de leyes naturales.

Cabe señalar que por explicación aquí se está entendiendo algo muy parecido a la intuición detrás del modelo nomológico-deductivo de la explicación. Según éste, una explicación consiste de una relación lógica entre algo a ser explicado y algo que explica. Esta relación lógica consiste en una implicación que va de lo que explica (enunciados generales y condiciones generales) hacia lo que se va a explicar (un enunciado menos general o particular). Así, si buscamos la explicación de las regularidades nómicas, entonces ésta debería ser implicada por algún enunciado más general y algunas condiciones generales. ¿Qué podría ser este o estos enunciados más generales de los que se deriven las regularidades a explicar? Pues aquellos que refieran a regularidades nómicas o las leyes naturales. ${ }^{4}$

4 Esto no quiere decir que el autor del manuscrito desconozca otros modelos de la explicación o que no esté consciente de sus limitaciones. Sólo está aclarando en qué sentido hay que entender "explicación" para poder alcanzar la conclusión que pretende. Un sentido compartido por los autores que han seguido previamente esta estrategia. 
También es pertinente remarcar que algunos filósofos estarían inclinados en pensar que las inferencias inductivas, es decir, el proceso inferencial inductivo que los sujetos llevamos a cabo puede brindarnos una explicación de las regularidades en la medida en que éstas son las responsables o el medio por el que nos topamos con ellas. Sin embargo, aunque es verdad que a través de esta herramienta llegamos a verdades generales a partir de una colección de hechos particulares observados sucesivamente similares entre sí, no parece que ésta explique por qué los objetos de un determinado tipo (natural o no) tienen las propiedades que tienen o por qué se comportan de la manera en que lo hacen. Para ilustrar esto, piénsese en (d). Esta verdad parecería ser el resultado de un tipo de enumeración como la siguiente "el cuerpo $x$ es un planeta que se mueve en una órbita elíptica y tiene al Sol como uno de sus focos; el cuerpo $y$ es un planeta que se mueve en una órbita elíptica y tiene al Sol como uno de sus focos; el objeto $z \ldots$ y así sucesivamente. Incluso si este procedimiento fue de hecho seguido por científicos como Kepler para llegar al conocimiento de regularidades nómicas acerca de los planetas, "saber cómo" no es lo mismo que "saber por qué". Este último tipo de conocimiento es el que perseguimos cuando tenemos como objetivo explicar las regularidades nómicas en las ciencias naturales. En breve, estamos partiendo de la idea de que una explicación tiene que responder satisfactoriamente la pregunta ¿por qué? en vez de la pregunta ¿cómo? Por consiguiente, para responder la primera pregunta uno está buscando razones o la justificación de la creencia, mientras que para responder la segunda uno está buscando algún tipo de descripción del procedimiento llevado a cabo o la herramienta utilizada para obtener tal creencia. Incluso si tuviésemos una teoría de cómo los científicos llegan a inferir que hay regularidades nómicas, ésta no sería capaz de explicarlas. Esto es así porque estamos interesados en la estructura y naturaleza del mundo físico y no en la estructura o naturaleza de nuestros procesos cognitivos.

A partir de todas las consideraciones hechas hasta aquí se llega a la conclusión de que las regularidades nómicas son explicadas por leyes naturales. Tal conclusión, insisto, sólo será consecuencia de aceptar el PRS, principio que es aceptable tanto por empiristas y antirrealistas por igual, y el principio que tiene que ver con privilegiar la explicación sin importar que esté formulada en términos de inobservables, un principio que el empirista no aceptará, así como de excluir otras alternativas de explicación tales como las dadas en términos de las regularidades mismas o sus instanciaciones y las hechas en términos de potencias o propensiones como explicaciones genuinas. 


\section{Un paso ulterior}

Al realista científico en general parece razonable pensar que, si algo genuinamente explica, entonces muy probablemente exista objetivamente. Esto es una forma de caracterizar la inferencia eliminativa o a la mejor explicación (IME) que Harman (1966, p. 89) describió como el proceso por el cual uno infiere, a partir del hecho de que una determinada hipótesis explicaría la evidencia, la verdad de dicha hipótesis. En el contexto del debate sobre cómo interpretar las teorías científicas actuales, el realista infiere a partir del éxito explicativo y predictivo de éstas que la mayoría de las entidades postuladas en ellas muy probablemente existan objetivamente. A esta inferencia se le conoce como "El argumento de los no milagros" (ANM) y su formulación primigenia fue dada por Putnam (1984). En el contexto particular que nos atañe, el realista infiere, a partir del hecho de que sólo las leyes naturales explican las regularidades, que las leyes naturales existen. Esta inferencia hecha por los realistas es denominada por Mumford (2004) "El argumento nomológico" (AN).

Cabe señalar que desde la lógica deductiva este tipo de inferencias carece de justificación o son inválidas. La razón de esto estriba en que no hay una regla de inferencia que nos permita establecer la verdad del antecedente a partir de la verdad del consecuente. En otras palabras, desde la lógica deductiva, al hacer esto estamos frente al argumento inválido denominado la afirmación del consecuente. Empero, reducir la inferencia eliminativa o la IME a un argumento deductivo inválido es pasar por alto que este tipo de inferencia es el medio por el cual los científicos formulan hipótesis desde cierta evidencia observada descartándolas poco a poco hasta quedarse con aquella que mejor explique dicha evidencia. En consecuencia, hay autores que arguyen que la inferencia eliminativa o a la mejor explicación puede justificarse desde su carácter confiable. La justificación de la inferencia eliminativa o IME en términos de su confiabilidad ha sido puesta sobre la mesa por autores tales como Harman (1965), Psillos (1996) y Lipton (1999). Este último menciona como ejemplo paradigmático de la confiabilidad de este tipo de inferencias la postulación de la existencia de Neptuno por parte de Adams y Leverrier como la mejor explicación de la perturbación de la órbita de Urano. En este ejemplo, ambos científicos, infirieron, a partir de las perturbaciones bien conocidas en las órbitas de Urano, la existencia de un planeta nuevo al que denominaron Neptuno. Algunos autores como Van Fraassen (1980) arguyen que la IME no conduce a la verdad de la hipótesis seleccionada como la que mejor explica la evidencia observada, pues puede ser el caso que dentro del conjunto de hipótesis que explican la evidencia ninguna de 
hecho sea verdadera. Por lo tanto, Van Fraassen remarca que pensar lo contrario, como lo hacen los partidarios de la IME, es producto de asumir que los seres humanos tenemos un tipo de privilegio al formular las hipótesis. Esta línea de argumentación es similar a la que Hanson (1961) formuló cuando afirmaba que la abducción Pierciana o IME sólo nos da razones para sugerir una hipótesis como la mejor de entre otras, pero que nunca daba razones para aceptar tal hipótesis seleccionada. Para lograr esto último se requiere suponerla como verdadera y llevar a cabo pruebas empíricas derivando predicciones observables a partir de ella (Niiniluoto 1999, p. 441). A pesar de estas objeciones a la IME, Niiniluoto (1999) arguye que es más prometedor sostener que la IME es en gran medida confiable dentro de nuestra vida cotidiana y que este hecho de la vida humana se puede explicar dando una razón evolutiva naturalista que consiste en la tesis de que todo el conocimiento humano no es sino el desarrollo de nuestros instintos animales innatos tal y como, según él, hizo Peirce.

Regresando a nuestro asunto, uno podría justificar la inferencia de que las leyes naturales existen a partir de que sólo ellas explican las regularidades de una manera análoga a la empleada por los partidarios del realismo científico cuando justifican la inferencia de que determinados procesos o entidades postulados por una teoría existen a partir del hecho de que éstos resultan indispensables para explicar y predecir determinados fenómenos observables.

Aun así, parece todavía que no hemos cerrado la puerta a una forma de antirrealismo. Esta afirmaría que del hecho de que una entidad o proceso postulado por una teoría científica se vuelve indispensable en la explicación de fenómenos observables no se sigue que éste exista objetivamente, pues muy bien puede tener una existencia meramente de orden conceptual o categórica de tal manera que sólo nos permita ordenar nuestras experiencias 5 . De la misma manera, en el caso de las leyes, este tipo de antirrealismo argüiría que éstas podrían no existir objetivamente, sino únicamente en tanto categorías o artilugios conceptuales que permitan reconocer regularidades a partir de la observación sucesiva de hechos particulares de un mismo tipo. No resulta sostenible pensar las leyes naturales como meras categorías o artilugios conceptuales dado que concebirlas de esta manera implica negar que hay un fundamento ontológico

\footnotetext{
5 Esta forma de pensar sobre cualquier término que encontremos en las teorías científicas es socavaba con la tesis como la de Hacking (1983) de que los científicos tienen certeza de que un término teórico tiene referente físico externo hasta el momento en que se emplea la entidad a la que refiere dicho término en algún arreglo experimental que nos permita inferir la existencia de otro proceso o entidad hasta cierto punto independiente.
} 
(universales, propensiones, potencias, etc.) que permita trazar la distinción entre leyes naturales y regularidades, sólo haciendo esta distinción se pueden caracterizar las primeras como explicando las segundas.

Por último, no se está afirmando aquí que aceptar el PRS y un tipo de realismo cíentífico, a saber, el de entidades, nos lleve a suscribir un realismo fuerte de leyes naturales. Más bien se está argumentado que a partir del hecho de que hay regularidades y la suposición de que estas requieren explicación, supuesto que de entrada los partidarios del empirismo constructuctivo pondrán en tela de juicio, ésta se puede dar en términos de ellas mismas (explicación que aceptarían los empiristas, dado que estaría formulada en términos de observables) o leyes naturales (explicación que sólo aceptarían los realistas científicos, dado que privilegian la explicación en términos de observables e inobservables por igual). La explicación en términos de ellas mismas no es una explicación genuina. La explicación en términos de disposiciones, propensiones o potencias sugerida por Mumford (2004) se identifica con la manera en que Bird (2005) caracteriza las leyes naturales y tal explicación, en tanto sugiere eliminar las leyes naturales de nuestra ontología, presenta las mismas dificultades que se ha denunciado presenta la forma de caracterizarlas por Armstrong (1983), Dretske (1977) y Tooley (1977). Por lo tanto, al no haber buenas razones para eliminar las leyes de nuestra ontología, sólo éstas, definidas disposicionalmente o en términos de universales, explican genuinamente las regularidades. Luego, se arguye que del hecho de que las leyes naturales sean explicación genuina de las regularidades se podría justificar su existencia objetiva. Esta inferencia, sólo estarían justificados en hacerla los partidarios del realismo científico de la misma manera en que creen están justificados inferir la verdad de las teorías científicas y/o la existencia objetiva de algunas de las entidades que éstas postulan a partir de su éxito explicativo y predictivo.

\section{Conclusión}

Parece que tenemos un argumento válido. La validez del argumento sería asentida, en principio, por todos los partidarios del realismo científico, salvo aquellos que simpaticen con algún tipo de eliminativismo y en el fondo con algunos supuestos de corte empirista, no así para todos los partidarios del antirrealismo en general. Esto es así por una falta de suposiciones compartidas entre ambos referentes a los aspectos metafísicos del realismo. Uno de estos aspectos tiene que ver con el privilegio de la explicación en términos observables e inobservables. Mientras que el antirrealista alega que el PRS no 
debería suscribirse en el caso del éxito de nuestras mejores teorías científicas actuales ni en el caso de las regularidades nómicas, el realista considera que ambos requieren explicación. Aún si el antirrealista aceptase el PRS y, por consiguiente, asienta que las regularidades tienen explicación, tal explicación no podría ser en términos de leyes naturales debido a que son escépticos de la existencia de conexiones necesarias. Esto es así porque tal conexión, arguyen, no es observada directamente por nuestras capacidades sensoriales, sino más bien es una idea que se infiere injustificadamente a partir de observar una sucesión de hechos particulares del mismo tipo. El realista, por el contrario, aceptará la postulación dichas entidades metafísicas, es decir, no observables en principio, en la medida en que tengan una función explicativa. Al privilegiar la explicación sin importar que este formulada en términos de observables e inobservables, el realista aceptaría el argumento presentado aquí como válido, siempre y cuando no simpatice con la idea de que las leyes naturales puedan caracterizarse como regularidades, mientras que el antirrealista al restringir la explicación exclusivamente a entidades observables, no aceptará la conclusión del argumento. En consecuencia, el argumento aquí presentado debería convencer solamente a los filósofos que se consideran a sí mismos como realistas científicos acerca de las teorías, pero que no han puesto la atención suficiente a las consecuencias relacionadas con la discusión acerca de las leyes naturales, de que hay buenas razones para creer que las leyes naturales existen objetivamente si se les caracteriza en términos de conexiones, vínculos o relaciones necesarias, en la medida en que i) esta creencia posibilita una explicación genuina de las regularidades y ii) esta creencia está justificada de la misma manera en que está justificado creer en la existencia objetiva de entidades o procesos inobservables postulados por las teorías en tanto dicha creencia explica su éxito predictivo y explicativo, en tanto estas inferencias son casos de la IME y ésta tiene su justificación en su carácter confiable. Si esto es así, no cabe duda de que hay una implicación indirecta entre el realismo acerca de las teorías científicas y el realismo de las leyes naturales. En otras palabras, la discusión acerca de las leyes naturales no es una cuestión aislada de la discusión entre realistas y antirrealistas acerca de las teorías científicas. 


\section{Bibliografía}

Armstrong, David (1983). What is a law of nature. Cambridge: Cambridge University Press.

Barnes, Eric (1994). "Explaining Brute Facts". PSA: Proceedings of the Biennial Meeting of the Philosophy of Science Association, vol. 1994, Volume One: Contributed Papers, 1994, pp. 61-68.

Bird, Alexander (2007). "Are there any laws, and if so what are they?". Laws and Properties, Clarendon, Oxford University Press, pp. 189-203.

- (2005). "The Dispositionalist Conception of Laws". Foundations of Science, 10, pp. 353-370.

— (1998). Philosophy of Science. Londres, Routledge, UCL Press.

Berenstain, Nora; Ladyman, James (2012). "Ontic Structural Realism and Modality”. En: Elaine Landry \& Dean Rickles (eds.), Structural Realism: Structure, Object and Causality.

Borge, Bruno (2016a). "Realism about What? Unobservable Entities and the Metaphysics of Modality”. Unisinos Journal of Philosophy, vol.17/1, pp. 69-74.

- (2016b). "Sobre la relación entre los realismos científico, modal y nomológico". Kriterion: Revista de Filosofía, vol. 57/135.

- (2015). "Sobre la crítica de Mumford al realismo nomológico". Manuscrito: Revista Internacional de Filosofía, vol. 38/3, pp. 59-80.

Carroll, John (1994). Laws of Nature. Cambridge: Cambridge University Press.

Chakravartty, Anjan (2017). Scientific Ontology: Integrating naturalized metaphysics and voluntarist epistemology, Oxford Studies in Philosophy of Science, Nueva York, Oxford University Press.

- (2007). A Metaphysics for Scientific Realism. Nueva York, Cambridge University Press.

Dretske, Fred (1977). "Laws of Nature”. Philosophy of Science, vol. 44/2, pp. 248268.

Fahrbach, Ludwig (2005). “Understanding Brute Facts”. Synthese, vol. 145/3, pp. 449-466.

Friedman, Michael (1974). "Explanation and Scientific Understanding". The Journal of Philosophy, vol. 71/1, pp. 5-19.

Harman, Gilbert (1965). "The Inference to the Best Explanation”. The Philosophical Review, vol. 74/1, pp. 88-95. 
Hacking, IAn (1983). Representing and Intervening. Cambridge, Cambridge University Press.

Kitcher, Philip (1983). "Explanatory Unification”. Philosophy of Science, vol. 48/4, pp. $507-531$.

Laudan, Larry (1981). "A Confutation of Convergent Realism". Philosophy of Science, vol. 48/1, pp. 19-49.

Lewis, David (1973). Counterfactuals. Cambridge: Harvard University Press.

Lipton, Peter (1991). Inference to the best explanation. Londres: Routledge.

Mumford, Stephen (2004). Laws in Nature. Londres: Routledge.

Nirniluoto, Ilkka (1999). "Defending Abduction”. Philosophy of Science, vol. 66, Proceedings of the 1998 Biennial Meetings of the Philosophy of Science Association. Part I: Contributed Papers (Sep. 1999), pp. 436-451.

Psillos, Stathis (1996). "In defence of scientific realism”. Scientific Realism: how science tracks truth, London: Routledge, pp. 68-94.

- (2014). "Regularities, Natural Patterns and Laws of Nature". Theoria: Revista de Teoría, Historia y Fundamentos de la Ciencia, vol. 29/1, pp. 9-27.

Tooley, Michael (1977). “The Nature of Laws”. Canadian Journal of Philosophy, vol. 7/4, pp. 667-698.

Van Fraassen, BaAs (1980). “Inference to the Best Explanation: Salvation by Laws?". The Scientific Image, Nueva York: Oxford University Press, pp. 131-150.

Walton, Douglas (1985). "Are circular arguments necessarily vicious?”. American Philosophical Quarterly, vol. 22/4, pp. 263-274.

Woodward, James; Walton, Douglas (1975). "Petitio Principii”. Synthese, vol. 31/1, pp. $107-127$.

Enviado: 26/05/2017

Recibido: 8/12/2017

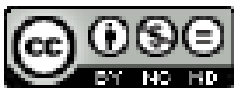

ENDOXA está bajo una licencia de Creative Commons Reconocimiento-NoComercial-SinObraDerivada 4.0 Internacional 
